\title{
Discrete Multiwavelet Critical-Sampling Transform-Based OFDM System over Rayleigh Fading Channels
}

\author{
Sameer A. Dawood, ${ }^{1}$ F. Malek, ${ }^{2}$ M. S. Anuar, ${ }^{1}$ and Suha Q. Hadi ${ }^{1}$ \\ ${ }^{1}$ School of Computer and Communication Engineering, University Malaysia Perlis (UniMAP), 02000 Arau, Perlis, Malaysia \\ ${ }^{2}$ School of Electrical Systems Engineering, University Malaysia Perlis (UniMAP), 02000 Arau, Perlis, Malaysia \\ Correspondence should be addressed to Sameer A. Dawood; sameer.ad80@yahoo.com
}

Received 31 December 2014; Accepted 10 May 2015

Academic Editor: Lotfi Senhadji

Copyright ( 2015 Sameer A. Dawood et al. This is an open access article distributed under the Creative Commons Attribution License, which permits unrestricted use, distribution, and reproduction in any medium, provided the original work is properly cited.

Discrete multiwavelet critical-sampling transform (DMWCST) has been proposed instead of fast Fourier transform (FFT) in the realization of the orthogonal frequency division multiplexing (OFDM) system. The proposed structure further reduces the level of interference and improves the bandwidth efficiency through the elimination of the cyclic prefix due to the good orthogonality and time-frequency localization properties of the multiwavelet transform. The proposed system was simulated using MATLAB to allow various parameters of the system to be varied and tested. The performance of DMWCST-based OFDM (DMWCSTOFDM) was compared with that of the discrete wavelet transform-based OFDM (DWT-OFDM) and the traditional FFT-based OFDM (FFT-OFDM) over flat fading and frequency-selective fading channels. Results obtained indicate that the performance of the proposed DMWCST-OFDM system achieves significant improvement compared to those of DWT-OFDM and FFT-OFDM systems. DMWCST improves the performance of the OFDM system by a factor of $1.5-2.5 \mathrm{~dB}$ and $13-15.5 \mathrm{~dB}$ compared with the DWT and FFT, respectively. Therefore the proposed system offers higher data rate in wireless mobile communications.

\section{Introduction}

One of the appealing multicarrier modulation schemes to accomplish the requirement of high data rate is orthogonal frequency division multiplexing (OFDM). The OFDM system divides the high data rate stream into a number of lower rate streams that are transmitted together over a number of orthogonal subcarriers to achieve frequency flat fading [1]. However, in wireless communication systems, the dependability of OFDM is restricted because of the time-varying characteristics of the channel, which causes intersymbol interference (ISI) and intercarrier interference (ICI). ISI and ICI can be averted effectively by inserting a cyclic prefix (CP) before each block of OFDM data symbols. However, CP introduces a loss in transmission power and reduction in the bandwidth efficiency $[2,3]$.

Inverse fast Fourier transform (IFFT) and fast Fourier transform (FFT) are normally used in the implementation of OFDM systems to create and detect the different orthogonal subcarriers. Although these transforms reduce the implementation complexity and are more computationally efficient, they have drawbacks that create rather high side lobes due to the use of a rectangular window. Moreover, the pulse shaping function used to modulate each subcarrier extends to infinity in the frequency domain, which leads to high interference and lower performance levels $[4,5]$.

Moreover, one major problem of the FFT-based OFDM (FFT-OFDM) system is the high peak-to-average power ratio (PAPR), which causes intermodulation distortion in the transmitted signal $[6,7]$.

Given the weak points of the FFT-OFDM system, many researchers have examined the use of wavelet-based OFDM to substitute Fourier-based OFDM; they found that the former has more advantages than the Fourier-based OFDM [8-11]. In OFDM based on wavelet transform, the IFFT and FFT blocks are merely replaced by inverse discrete wavelet transform (IDWT) and discrete wavelet transform (DWT), respectively. Wavelet transform offers much lower side lobes in the transmitted signal, which reduces its sensitivity to ICI. The most significant difference between FFT-OFDM and 
DWT-based OFDM (DWT-OFDM) is that the FFT-OFDM signals only overlap in the frequency domain, whereas the DWT-OFDM signals overlap in both time and frequency domains. The time overlap in the DWT-OFDM system allows the system to exclude the use of $\mathrm{CP}$ or any kind of guard interval (GI) that is usually used in FFT-OFDM system. Hence, the spectral containment of the channel in DWT-OFDM is better as it does not use CP. A previous research [12] investigated the performance of the OFDM system based on a wavelet with different families, such as Haar, Daubechies, biorthogonal, and reverse biorthogonal wavelets. They found that the Haar wavelet provides a very good platform for wireless communication with minimum bit error rate (BER), ISI, and PAPR. In a previous study [13], performance comparisons of FFT-OFDM and DWT-OFDM were conducted using different types of wavelet transform, such as Haar, Daubechies, and biorthogonal wavelets. The DWT-OFDM system is better compared with the FFTOFDM scheme under certain channel conditions. Another research [14] investigated the performance of DWT-OFDM against FFT-OFDM in terms of PAPR. The DWT-OFDM gives a reduction of $1.63 \mathrm{~dB}$ compared with the FFT-OFDM system.

More performance gains can be achieved by looking at substitute orthogonal base functions and finding a better transform compared with wavelet and Fourier transforms. Multiwavelet is really a new section that has been added to wavelet theory recently [15-17]. It has multiple scaling and wavelet functions in each level rather than one scaling function and one wavelet function in wavelet transform. This setup means a greater degree of freedom in constructing wavelets. Therefore, in contrast to scalar wavelet, properties such as compact support, orthogonality, symmetry, vanishing moments, and short support can be gathered simultaneously in multiwavelet, which is essential in signal processing. All the properties of multiwavelet transform are suitable for application in OFDM systems.

The authors of a previous study [18] proposed an OFDM system based on discrete multiwavelet transform (DMWT) with oversampling preprocessing. The BER performance of the proposed system was simulated for different channel models. They found that the oversampling DMWT-OFDM system achieved much lower BER and better performance than DWT-OFDM and FFT-OFDM under AWGN, flat fading, and frequency-selective fading channels. In oversampling preprocessing, the input data is repeated with the same data multiplied by a constant. Oversampling preprocessing doubles the input data symbols, which reduces the bandwidth efficiency substantially; it also increases the computational complexity of the transform. The implementation of WiMAX (IEEE802.16d) based on oversampling DMWT-OFDM over wireless communications channels was presented in [19]. The proposed design achieved much lower BER and robustness for multipath channels and did not require $\mathrm{CP}$, which indicates that it has higher spectral efficiency than OFDM based on DWT and FFT. In [20], the design and performance of HIPERLAN/2 standard model were improved using oversampling DMWT. The proposed design achieved much lower BER and better performance than traditional system based on FFT in different channel models.

In this paper, discrete multiwavelet critical-sampling transform (DMWCST) is proposed for OFDM systems to achieve better BER performance than conventional OFDM using FFT and DWT over flat fading and frequency-selective fading channels. The proposed DMWCST maintains the same data rate of input symbols, which increases the bandwidth efficiency and reduces the computational complexity. The proposed DMWCST-OFDM system will be presented depending on a fast computation algorithm for DMWCST.

The rest of the paper is arranged as follows. Section 2 presents the background of the discrete multiwavelet criticalsampling transform. Section 3 presents the proposed system. Section 4 discusses the simulation results. Section 5 presents the computational complexity analysis, and Section 6 presents our conclusions.

\section{Discrete Multiwavelet Critical-Sampling Transform (DMWCST)}

The theory of multiwavelet is based on the idea of multiresolution analysis (MRA) similar to that in the scalar wavelet. The difference is that multiwavelets have several scaling and wavelet functions. Multiwavelets have several advantages in comparison to scalar wavelets. Features such as short support, orthogonality, symmetry, and vanishing moments are important in signal processing. A scalar wavelet cannot possess all these properties at the same time. Thus, multiwavelets offer the possibility of superior performance for image processing applications compared with scalar wavelets [21]. The multiwavelets studied to date consist of two scaling functions and two wavelet functions. Multiwavelet scaling functions $\{\phi(t)\}$ and wavelet functions $\{\psi(t)\}$ can be represented by the following equations $[15,17,21]$ :

$$
\begin{aligned}
& \phi(t)=\sqrt{2} \sum_{k=-\infty}^{\infty} H_{k} \phi(2 t-k), \\
& \psi(t)=\sqrt{2} \sum_{k=-\infty}^{\infty} G_{k} \phi(2 t-k),
\end{aligned}
$$

where $H_{k}$ and $G_{k}$ are the filter coefficients of scaling and wavelet functions, respectively. Both $H_{k}$ and $G_{k}$ are $2 \times 2$ matrices for each integer $k$. The value of $\sqrt{2}$ maintains the norm of the scaling and wavelet functions with a scale of two.

Equations (1) can be implemented as a matrix filter bank as seen in Figure 1, resulting in two channels operating on two input data streams. They are then filtered into four output data streams, each of which is downsampled by a factor of two. Blocks $H$ and $G$ are low- and high-pass analysis filters, and $\widetilde{H}$ and $\widetilde{G}$ are low- and high-pass synthesis filters [21].

Geronimo, Hardian, and Massopust suggested a useful multiwavelet filter known as GHM. GHM filter offers a mixture of orthogonality, symmetry, and compact support, which are important in signal processing [15]. In the GHM system, $H_{k}$ consists of four scaling matrices, namely, $H_{0}$, 


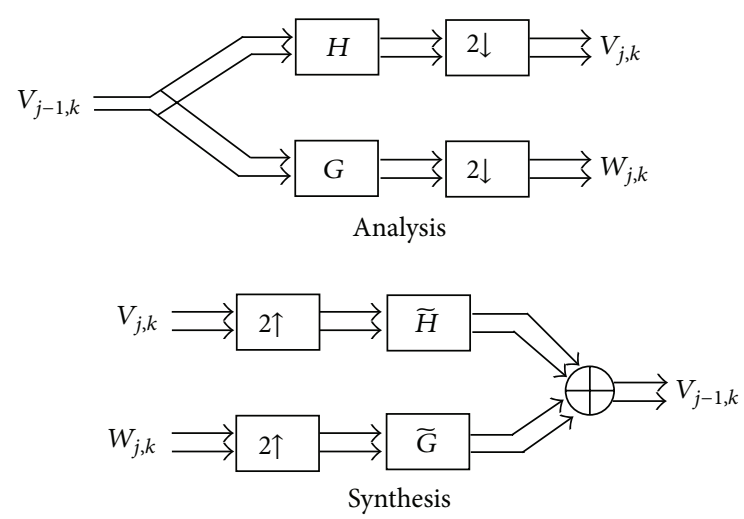

FIGURE 1: Analysis and synthesis stages of 1D single-level multiwavelet transform.

$H_{1}, H_{2}$, and $H_{3}$, as given in (2). $G_{k}$ consists of four wavelet matrices, namely, $G_{0}, G_{1}, G_{2}$, and $G_{3}$, as given in (3) [15]. Consider the following:

$$
\begin{aligned}
& H_{0}=\left[\begin{array}{cc}
\frac{3}{5 \sqrt{2}} & \frac{4}{5} \\
\frac{-1}{20} & \frac{-3}{10 \sqrt{2}}
\end{array}\right] \text {, } \\
& H_{1}=\left[\begin{array}{cc}
\frac{3}{5 \sqrt{2}} & 0 \\
\frac{9}{20} & \frac{1}{\sqrt{2}}
\end{array}\right] \text {, } \\
& H_{2}=\left[\begin{array}{cc}
0 & 0 \\
\frac{9}{20} & \frac{-3}{10 \sqrt{2}}
\end{array}\right] \text {, } \\
& H_{3}=\left[\begin{array}{cc}
0 & 0 \\
\frac{-1}{20} & 0
\end{array}\right] \\
& G_{0}=\left[\begin{array}{cc}
\frac{-1}{20} & \frac{-3}{10 \sqrt{2}} \\
\frac{1}{10 \sqrt{2}} & \frac{3}{10}
\end{array}\right] \text {, } \\
& G_{1}=\left[\begin{array}{cc}
\frac{9}{20} & \frac{-1}{\sqrt{2}} \\
\frac{-9}{10 \sqrt{2}} & 0
\end{array}\right] \text {, } \\
& G_{2}=\left[\begin{array}{cc}
\frac{9}{20} & \frac{-3}{10 \sqrt{2}} \\
\frac{9}{10 \sqrt{2}} & \frac{-3}{10}
\end{array}\right] \text {, } \\
& G_{3}=\left[\begin{array}{cc}
\frac{-1}{20} & 0 \\
\frac{-1}{10 \sqrt{2}} & 0
\end{array}\right] \text {. }
\end{aligned}
$$

Assume that the input sequence $f[n]$ contains samples of $f(t)$ at half-integers:

$$
\begin{aligned}
f[2 n] & =f(n), \\
f[2 n+1] & =f\left(n+\frac{1}{2}\right) .
\end{aligned}
$$



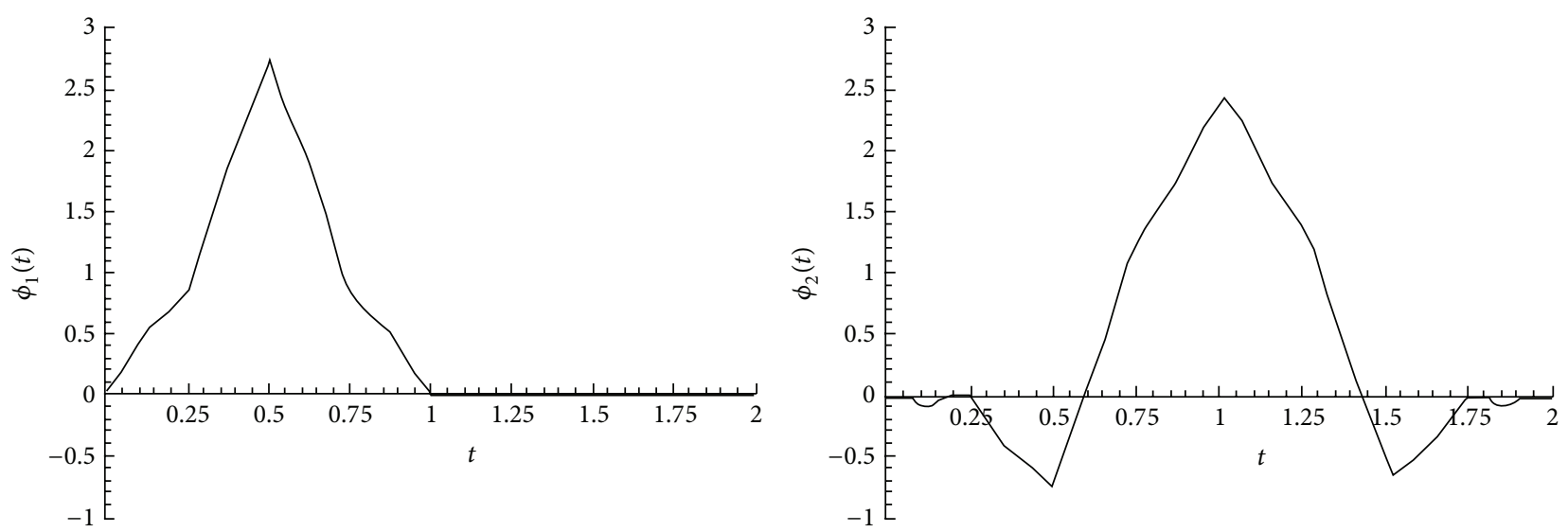

Figure 2: GHM pair of scaling functions [15].

As shown in Figure 2, $\phi_{1}(t)$ is zero at all integer points and $\phi_{2}(t)$ is nonzero value at integer 1 only. Sampling (5) at integers and half-integers gives [15]

$$
\begin{aligned}
f[2 n]= & \phi_{2}(1) v_{2, n-1}^{(0)}, \\
f[2 n+1]= & \phi_{2}\left(\frac{3}{2}\right) v_{2, n-1}^{(0)}+\phi_{1}\left(\frac{1}{2}\right) v_{1, n}^{(0)} \\
& +\phi_{2}\left(\frac{1}{2}\right) v_{2, n}^{(0)} .
\end{aligned}
$$

The coefficients $v_{1, n}^{(0)}, v_{2, n}^{(0)}$ can be easily found from (7) as follows:

$$
\begin{aligned}
& v_{1, n}^{(0)} \\
& =\frac{\phi_{2}(1) f[2 n+1]-\phi_{2}(1 / 2) f[2 n+2]-\phi_{2}(3 / 2) f[2 n]}{\phi_{2}(1) \phi_{1}(1 / 2)}, \\
& v_{2, n}^{(0)}=\frac{f[2 n+2]}{\phi_{2}(1)} .
\end{aligned}
$$

Taking into account the symmetry of $\phi_{2}(t),(8)$ can be written as follows:

$$
\begin{aligned}
& v_{1, n}^{(0)} \\
& =\frac{\phi_{2}(1) f[2 n+1]-\phi_{2}(1 / 2)(f[2 n+2]+f[2 n])}{\phi_{2}(1) \phi_{1}(1 / 2)}, \\
& v_{2, n}^{(0)}=\frac{f[2 n+2]}{\phi_{2}(1)} .
\end{aligned}
$$

Equations (9) offer a normal way to find two input rows $v_{1, n}^{(0)}, v_{2, n}^{(0)}$ which are generated from the original signal $f[n]$. Inverting (9), the signal in (7) can be recovered [15].
For any $1 \mathrm{D}$ signal $\left(X_{k}\right)$ of length $N \times 1$, where $N$ must be power of 2, (8) can be written as follows:

$$
\begin{aligned}
& X p_{k}=\frac{\phi_{2}(1) X_{k}-\phi_{2}(1 / 2) X_{k+1}-\phi_{2}(3 / 2) X_{k-1}}{\phi_{2}(1) \phi_{1}(1 / 2)} \\
& k=1,3,5, \ldots, N-1, \\
& X p_{k}=\frac{X_{k}}{\phi_{2}(1)}, \quad k=2,4,6, \ldots, N .
\end{aligned}
$$

Using GHM scaling function graph (Figure 2), the values for $\phi_{1}(1 / 2), \phi_{2}(1 / 2), \phi_{2}(3 / 2)$, and $\phi_{2}(1)$ should be found. Substituting these values in (10) results in the following:

$$
\begin{aligned}
& X p_{k}=(0.373615) X_{k}+(0.11086198) X_{k+1} \\
&+(0.11086198) X_{k-1}, \\
& \quad k=1,3,5, \ldots, N-1, \\
& X p_{k}=[\sqrt{2}-1] X_{k}, \quad k=2,4,6, \ldots, N .
\end{aligned}
$$

2.2. Matrix (Approximation) Method. In this method, the preprocessing of the input signal $\left\{X_{k}\right\}$ is achieved by splitting it in a sequence of $2 \times 1$ vectors $\left\{\left[\begin{array}{ll}X_{2(m+k)} & X_{2(m+k)+1}\end{array}\right]^{T}\right\}$ and applying the matrix prefilter $(P)$ (without downsampling) [23]:

$$
v_{j, k}=\sum_{m=0}^{M} P_{m}\left[\begin{array}{c}
X_{2(m+k)} \\
X_{2(m+k)+1}
\end{array}\right],
$$

where $P_{0}, P_{1}, \ldots, P_{M}$ are $2 \times 2$ matrix coefficients of $P$ and $M$ is the number of matrix prefilter coefficients. For the GHM system the following prefilter with two coefficients is usually used [23]:

$$
\begin{aligned}
& p_{0}=\left[\begin{array}{cc}
\frac{3}{8 \sqrt{2}} & \frac{10}{8 \sqrt{2}} \\
0 & 0
\end{array}\right], \\
& p_{1}=\left[\begin{array}{cc}
\frac{3}{8 \sqrt{2}} & 0 \\
1 & 0
\end{array}\right] .
\end{aligned}
$$


It preserves approximation of second orders. For secondorder approximation, (11) become

$$
\begin{aligned}
& X p_{k}=\left(\frac{10}{8 \sqrt{2}}\right) X_{k}+\left(\frac{3}{8 \sqrt{8}}\right) X_{k+1}+\left(\frac{3}{8 \sqrt{2}}\right) X_{k-1}, \\
& \quad k=1,3,5, \ldots, N-1, \\
& X p_{k}=X_{k}, \quad k=2,4,6, \ldots, N .
\end{aligned}
$$

It is clear from (11) for first-order approximation and (14) for second order approximation that the resulting signal after critically sampled preprocessing has the same length as before preprocessing. Hence, critically sampled preprocessing maintains the same data rate.

2.3. DMWCST Computation. Computation for single-level 1D DMWCST using fast algorithm can be accomplished through the following steps:

(1) The input signal $(X)$ is of length $N \times 1$, where $N$ should be power of 2 .

(2) The GHM filter coefficients given in (2) are used to generate the transformation matrix $\left(W_{1}\right)$ with size $N / 2 \times N / 2$, which is provided in (15). Since $H_{i}$ and $G_{i}$ are $2 \times 2$ matrices, an $N \times N$ transformation matrix is obtained after substituting the GHM filter coefficients in

$W_{1}$

$$
=\left[\begin{array}{ccccccccccc}
H_{0} & H_{1} & H_{2} & H_{3} & 0 & 0 & \cdots & 0 & 0 & 0 & 0 \\
0 & 0 & H_{0} & H_{1} & H_{2} & H_{3} & \cdots & 0 & 0 & 0 & 0 \\
\vdots & \vdots & \vdots & \vdots & \vdots & \vdots & \cdots & \vdots & \vdots & \vdots & \vdots \\
H_{2} & H_{3} & 0 & 0 & 0 & 0 & \cdots & 0 & 0 & H_{0} & H_{1} \\
G_{0} & G_{1} & G_{2} & G_{3} & \vdots & \vdots & \cdots & 0 & 0 & 0 & 0 \\
0 & 0 & G_{0} & G_{1} & G_{2} & G_{3} & \cdots & 0 & 0 & 0 & 0 \\
\vdots & \vdots & \vdots & \vdots & \vdots & \vdots & \cdots & \vdots & \vdots & \vdots & \vdots \\
0 & 0 & 0 & 0 & 0 & 0 & \cdots & G_{0} & G_{1} & G_{2} & G_{3} \\
G_{2} & G_{3} & 0 & 0 & 0 & 0 & \cdots & 0 & 0 & G_{0} & G_{1}
\end{array}\right] .
$$

(3) Preprocessing of the input signal $(X)$ through critically sampled preprocessing is achieved by applying (11) for first-order approximation or (14) for secondorder approximation to input signal to generate new signal $(X p)$.

(4) Transformation of input signal is accomplished through multiplying the $N \times N$ transformation matrix $\left(W_{1}\right)$ with the $N \times 1$ preprocessing input signal $(X p)$. Consider the following:

$$
[Y]_{N \times 1}=\left[W_{1}\right]_{N \times N} \cdot[X p]_{N \times 1} .
$$

Inverse discrete multiwavelet critical-sampling transform (IDMWCST) can be computed by the inverse of the upper procedure, as shown in the following steps:

(1) A multiwavelet transformed signal $(Y)$ of length $N \times 1$ exists.

(2) Generate a reconstruction matrix $\left(W_{2}\right)$, which is the inverse of the transformation matrix $\left(W_{1}\right)$ given in (15). $W_{1}$ is an orthogonal matrix, so its inverse is just the transposed

$$
\left[W_{2}\right]_{N \times N}=\left[W_{1}\right]_{N \times N}^{T} \text {. }
$$

(3) The reconstruction matrix $\left(W_{2}\right)$ is multiplied with the multiwavelet transformed signal $(Y)$ :

$$
[X p]_{N \times 1}=\left[W_{2}\right]_{N \times N} \cdot[Y]_{N \times 1} \cdot
$$

(4) Postprocessing is applied to $(X p)$ to find the original signal $(X)$ through the following equations:

(a) For first-order approximation,

$$
\begin{aligned}
& X_{k} \\
& =\frac{\left[X p_{k}-(0.11086198) X p_{k+1}-(0.11086198) X p_{k-1}\right]}{(0.373615)}, \\
& k=1,3,5, \ldots, N-1, \\
& X_{k}=\frac{X p_{k}}{[\sqrt{2}-1]}, \quad k=2,4,6, \ldots, N . \\
& \quad(b) \text { For second-order approximation, } \\
& X_{k}=\frac{\left[X p_{k}-(3 / 8 \sqrt{8}) X p_{k+1}-(3 / 8 \sqrt{2}) X p_{k-1}\right]}{(10 / 8 \sqrt{2})}, \quad k=1,3,5, \ldots, N-1, \\
& X_{k}=X p_{k}, \quad k=2,4,6, \ldots, N .
\end{aligned}
$$

\section{Proposed DMWCST-Based OFDM System}

Figure 3 symbolizes the complete model for the proposed DMWCST-OFDM system. The transmitter accepts serial binary data. The serial data are converted into low-rate sequences via serial-to-parallel (S/P) conversion and grouped and then mapped according to a mapping technique (quadrature phase shift keying (QPSK) was used in this work). The training sequence (pilot subcarriers) is then inserted to allow for channel estimation to be utilized to compensate for the channel effects of the required signal. The pilot carrier has a bipolar sequence $\{ \pm 1\}$. Now, the $N_{f}$-point IDMWCST based on second-order approximation presented in the previous section is applied to the signal to achieve the orthogonality between subcarriers. Zeros are inserted in several bins of IDMWCST to make the transmitted spectrum compact and reduce the adjacent carriers' interference. The addition of 


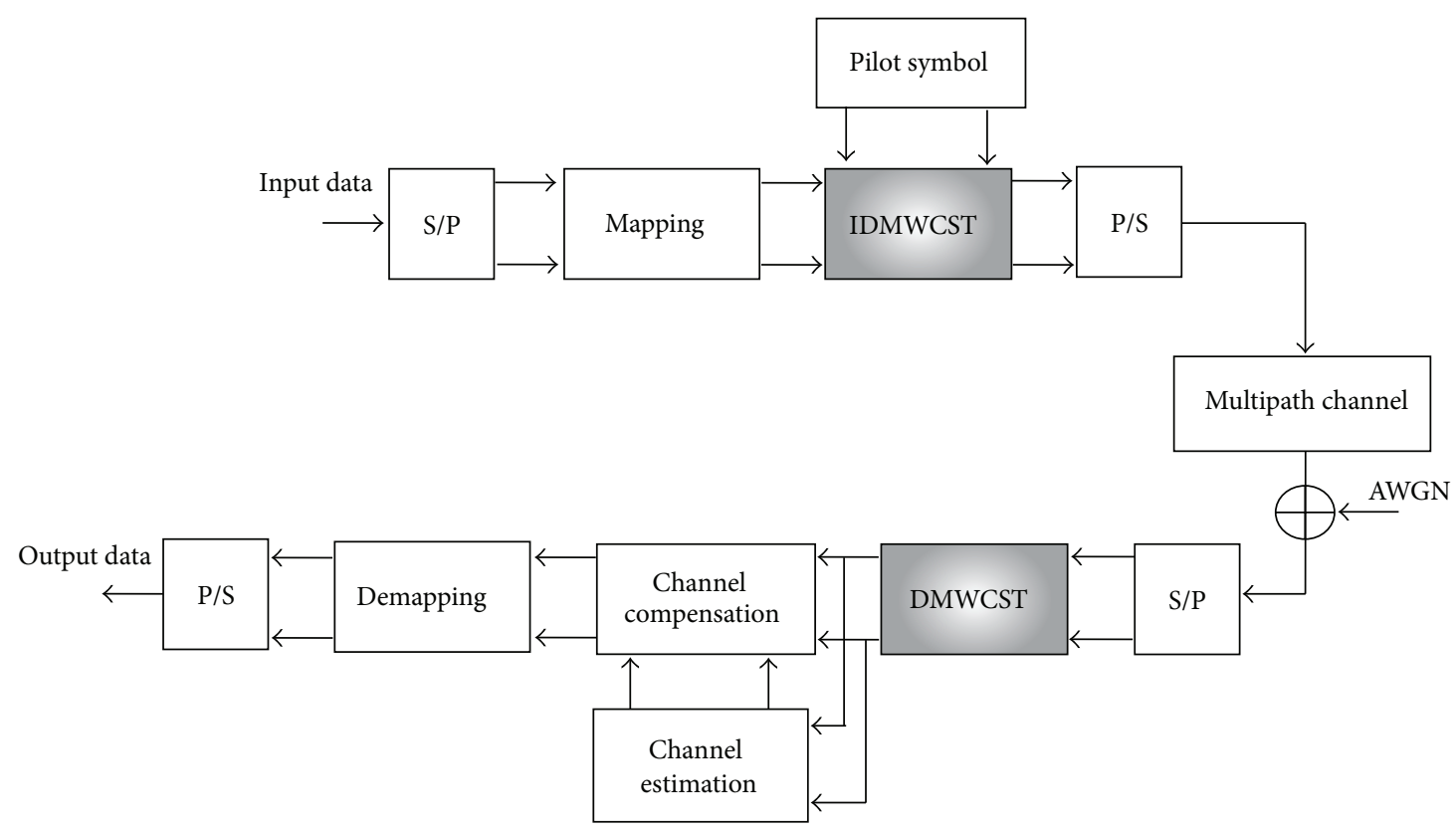

FIGURE 3: Block diagram of the proposed system.

zeros to some subcarriers means that not all the subcarriers are used; only the subset $\left(N_{c}\right)$ of total subcarriers $\left(N_{f}\right)$ is used. Finally, the parallel data are converted into serial via parallelto-serial $(\mathrm{P} / \mathrm{S})$ conversion and sent to the receiver over the wireless channel.

Given that CP is not added to OFDM symbols in the proposed system, the data rates in DMWCST-OFDM are higher than those in traditional FFT-OFDM.

The signal received from the wireless channel can be described as [24, 25]

$$
y(n)=x(n) * h(n)+w(n)
$$

where $y(n)$ is the received signal, $x(n)$ is the transmitted signal, $h(n)$ is the wireless channel impulse response, $w(n)$ is the additive white Gaussian noise (AWGN), and * refers to the convolution process.

At the receiver side, the inverse operations are performed in an opposite order to yield the correct data stream. The received signal is converted to a parallel version via $\mathrm{S} / \mathrm{P}$ conversion. $N_{f}$-point DMWCST based on second-order approximation is performed, and the zero pads are removed. The pilot subcarriers are then utilized to estimate the channel frequency response $(H(k))$ as follows:

$$
H(k)=\frac{Y_{p}(k)}{X_{p}(k)}, \quad k=1,2, \ldots, N_{c},
$$

where $Y_{p}(k)$ represents the received pilot subcarriers and $X_{p}(k)$ is the transmitted pilot subcarriers. The channel frequency response obtained in (22) is employed to compensate for the channel effects on the data. Estimated data $(\widehat{X}(k))$ can be obtained with the following equation:

$$
\widehat{X}(k)=H^{-1}(k) \cdot Y(k), \quad k=1,2, \ldots, N_{c} .
$$

Finally, the estimated data passes through the demapping technique to recover the original data. To calculate the BER, the received bits are compared to the transmitted bits for different values of signal-to-noise ratio (SNR).

\section{Simulation Results and Discussion}

The proposed DMWCST-OFDM system was simulated with MATLAB (version 7.8), and its BER performance was compared with that of DWT-OFDM and FFT-OFDM systems over flat fading and frequency-selective fading channels. Haar wavelet [26] was employed for DWT. The length of CP in FFT-OFDM was $25 \%$ of total symbol length of OFDM. The fading channel was considered a Rayleigh fading channel modeled as Jake's model [27]. Channel effect was assumed to be constant on each packet frame. Therefore, block-type pilot channel estimation [28] was employed. Table 1 shows the parameters and their values in the system utilized in the simulation.

Figure 4 shows the performance of the proposed system (DMWCST-OFDM) compared with that of DWT-OFDM and FFT-OFDM systems in flat fading channel according to the Doppler frequency ( $\mathrm{Fd}$ ) of $5 \mathrm{~Hz}$ (slow fading). In such case, all the frequency components in the signal will be affected by a constant attenuation and the linear-phase distortion of the channel. The DMWCST-OFDM performs much better than the DWT-OFDM and FFT-OFDM systems because the orthogonality between subcarriers in DMWCST is more significant than those in DWT and FFT. Clearly, for $\mathrm{BER}=10^{-3}$, the SNR for DMWCST-OFDM is about $16.8 \mathrm{~dB}$, whereas in DWT-OFDM, the SNR is about $19.2 \mathrm{~dB}$. Meanwhile, for FFT-OFDM, the SNR is about $31.9 \mathrm{~dB}$.

Figure 5 shows the BER performance of OFDM in flat fading channel according to $\mathrm{Fd}=200 \mathrm{~Hz}$ (fast fading). In the 
TABLE 1: Simulation parameters.

\begin{tabular}{lc}
\hline Parameter & Value \\
\hline System bandwidth & $10 \mathrm{MHz}$ \\
\hline Modulation type & QPSK \\
\hline $\begin{array}{l}\text { Number of FFT, DWT, and } \\
\text { DMWCST points }\left(N_{f}\right)\end{array}$ & 64 \\
\hline Number of useful subcarriers $\left(N_{c}\right)$ & 48 \\
\hline Channel type & $\begin{array}{c}\text { Flat fading + AWGN } \\
\text { Selective fading + AWGN }\end{array}$
\end{tabular}

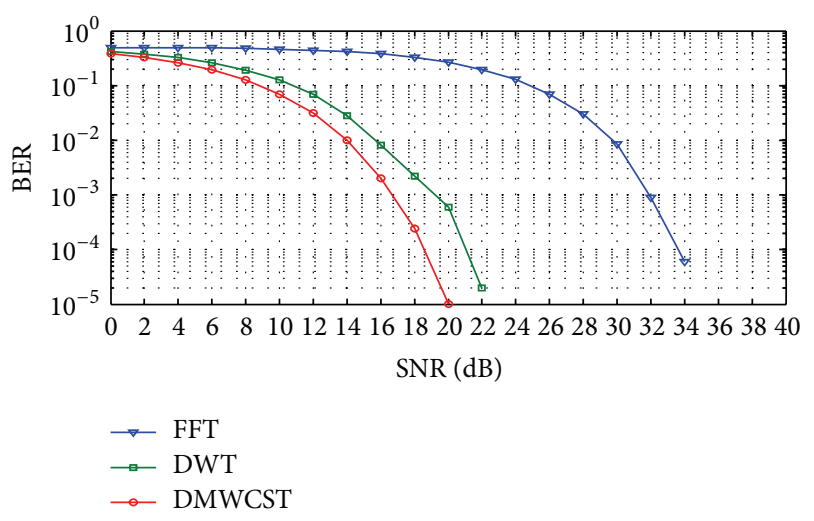

FIGURE 4: BER performance of OFDM system in the flat fading channel at $\mathrm{Fd}=5 \mathrm{~Hz}$.

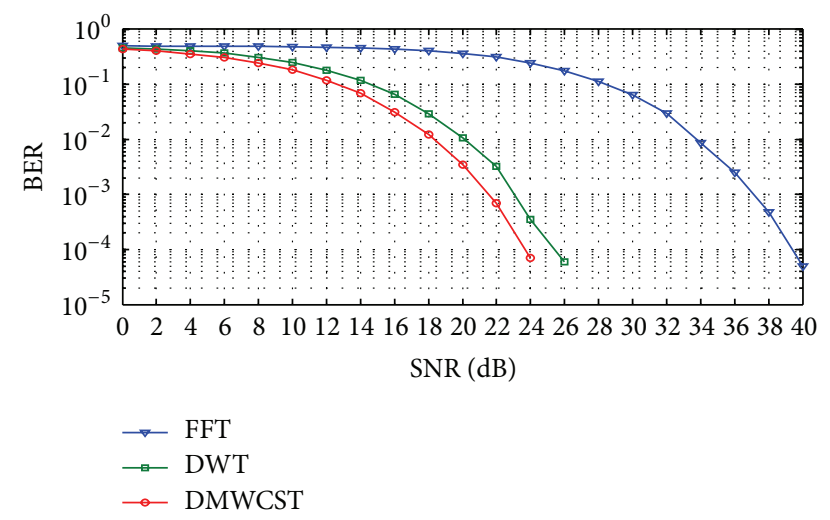

FIGURE 5: BER performance of OFDM system in the flat fading channel at $\mathrm{Fd}=200 \mathrm{~Hz}$.

fast-fading channel, BER increases for all schemes because the path gains of the channel vary in one frame (i.e., the coherence time of the fading channel is decreased). Based on this figure, the performance of the proposed system is superior to that of the other systems. The proposed system has BER of $10^{-3}$ at SNR $=21.5 \mathrm{~dB}$. DWT-OFDM has the same $\mathrm{BER}$ at $\mathrm{SNR}=23 \mathrm{~dB}$, and FFT-OFDM has the same BER at $\mathrm{SNR}=37 \mathrm{~dB}$.

Figure 6 gives the BER performance of OFDM in frequency-selective fading channel according to $\mathrm{Fd}=5 \mathrm{~Hz}$. Two paths were selected; the second path has a gain of $-10 \mathrm{~dB}$ and a delay of eight samples. As seen in this

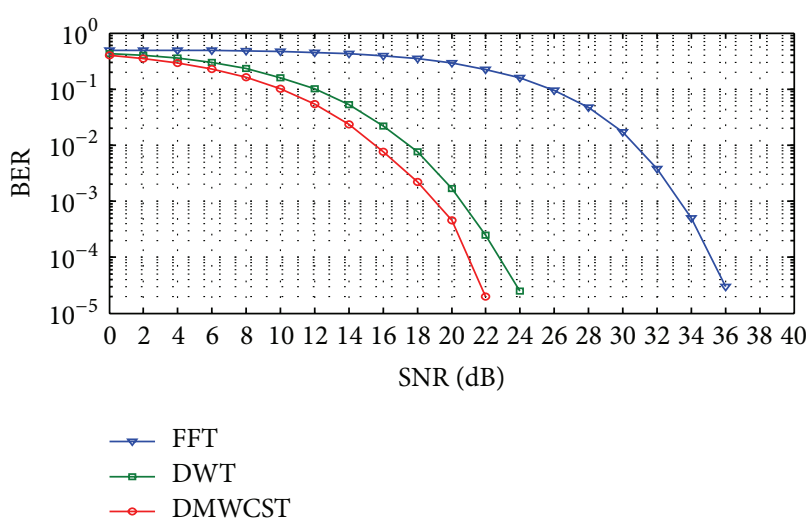

FIGURE 6: BER performance of OFDM system in the selective fading channel at $\mathrm{Fd}=5 \mathrm{~Hz}$.

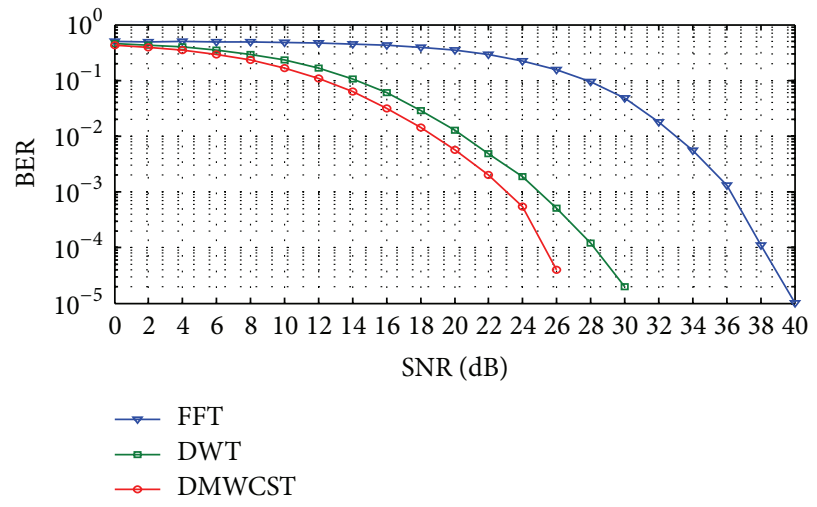

FIGURE 7: BER performance of OFDM system in the selective fading channel at $\mathrm{Fd}=200 \mathrm{~Hz}$.

figure, the proposed system is more robust in the frequencyselective fading channel compared with DWT-OFDM and FFT-OFDM. A BER $=10^{-3}$ resulted in $1.5 \mathrm{~dB}$ and $14.2 \mathrm{~dB}$ improvement for the proposed system compared with the DWT-OFDM and FFT-OFDM, respectively.

The comparison of the performance of the three systems in a frequency-selective fading channel according to $\mathrm{Fd}=$ $200 \mathrm{~Hz}$ is illustrated in Figure 7. Notably, the proposed system outperforms the DWT-OFDM by $2 \mathrm{~dB}$ and the FFT-OFDM by $13.1 \mathrm{~dB}$ at $\mathrm{BER}=10^{-3}$.

Figures 8 and 9 illustrate the effect of changing the second path gain on the performance of the proposed system. Two cases are studied, namely, $-5 \mathrm{~dB}$ and $-15 \mathrm{~dB}$, both at Doppler frequency of $5 \mathrm{~Hz}$. ISI will occur in the frequency-selective channel, and its magnitude will depend directly on the attenuation of the second path. Therefore, the ISI will increase with the increase of the attenuation, leading to an increase in BER. From these figures, the proposed system still outperforms the other two structures. As shown in Figure 8, BER $=10^{-3}$ results in 1.8 and $11.4 \mathrm{~dB}$ improvement for the DMWCSTOFDM compared with the DWT-OFDM and FFT-OFDM, respectively. In Figure 9, BER $=10^{-3}$ results in 1.3 and $15.25 \mathrm{~dB}$ improvement for the DMWCST-OFDM compared with the DWT-OFDM and FFT-OFDM, respectively. 
TABLE 2: Comparison of BER performance of DMWCST-OFDM and oversampling DMWT-OFDM [18].

\begin{tabular}{lccccc}
\hline & Fading channel & \multicolumn{2}{c}{ DMWCST-OFDM } & Oversampling DMWT-OFDM [18] \\
\hline BER & \multirow{2}{*}{ Flat } & $10^{-2}$ & $10^{-3}$ & $10^{-2}$ & $10^{-3}$ \\
SNR $(\mathrm{dB})$ & & 14 & 16.8 & 17 & 26 \\
\hline SNR $(\mathrm{dB})$ & Selective & 15.5 & 19 & 30 & - \\
\hline
\end{tabular}

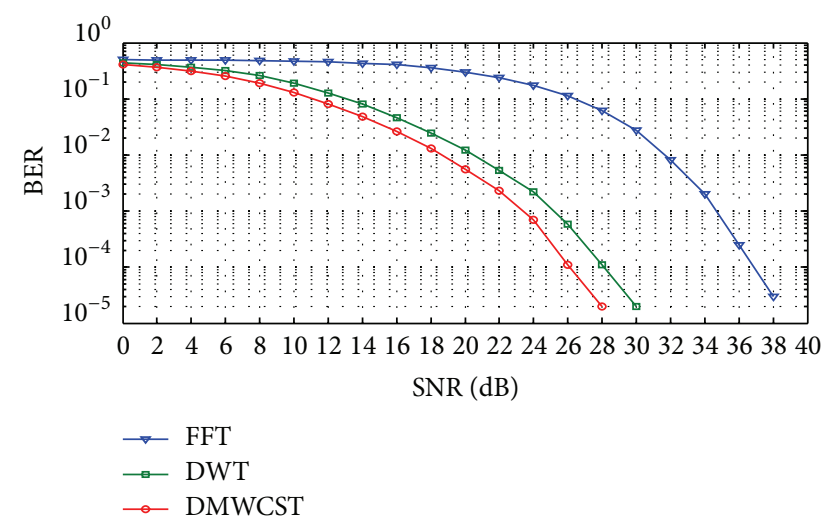

FIGURE 8: BER performance of OFDM system in the selective fading channel at second path gain $=-5 \mathrm{~dB}$.

The comparison between the proposed system and the system proposed by Kattoush et al. [18] can be shown in Table 2, in flat and frequency-selective fading channels at $\mathrm{Fd}=5 \mathrm{~Hz}$. From Table 2 , the proposed system reached $10^{-2}$ BER at $14 \mathrm{~dB}$ SNR, whereas [18] reached $10^{-2} \mathrm{BER}$ at $17 \mathrm{~dB}$ in flat fading channel. While in the frequency-selective fading channel the proposed system reached $10^{-2}$ BER at $15.5 \mathrm{~dB}$ SNR, [18] reached $10^{-2}$ BER at $30 \mathrm{~dB}$. Therefore, the proposed system outperforms the system proposed in [18].

\section{Computational Complexity Analysis}

In this section, the computational complexity of the DMWCST is analyzed and compared with the oversampling DMWT, DWT, and FFT. We consider here the case that all transforms of $N$-points make a fair comparison.

5.1. DMWCST. As shown in (15), the transformation matrix $\left(W_{1}\right)$ has many zeros; hence, its direct computation will only involve $8 \mathrm{~N}$ multiplications and $7 \mathrm{~N}$ additions. In the GHM filter, the presence of many zero coefficients and the linearphase symmetry can be exploited to reduce the computation complexity to $(17 / 4) N$ multiplications and (19/4) $N$ additions. It is worth mentioning that, for the case of complex constellation, the arithmetic operations must be calculated twice, one for the real part and the other for the imaginary part. Therefore the real multiplications $\left(R_{M}\right)$ will be equal to $(17 / 2) N$, and the real additions $\left(R_{A}\right)$ will be equal to $(19 / 2) N$.

5.2. Oversampling DMWT. In oversampling DMWT, the input data is repeated with the same data multiplied by a constant $[15,16]$. Oversampling preprocessing doubles

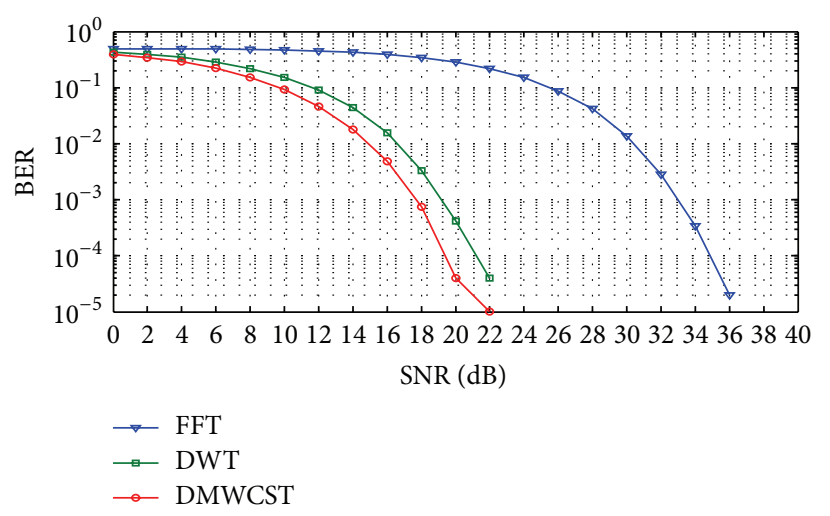

FIGURE 9: BER performance of OFDM system in the selective fading channel at second path gain $=-15 \mathrm{~dB}$.

the input data symbols; hence, the transformation matrix $\left(W_{1}\right)$ will be doubled in dimensions. Therefore, the computational complexity of the multiwavelet transform will be increased by a factor of two, so that the number of real multiplications will be $R_{M}=17 N$, and the number of real additions will be $R_{A}=19 N$.

5.3. DWT. DWT with a filter of length $(L)$ required $L N$ multiplications and $(L-1) N$ additions [29]. Same as in DMWCST, when the complex constellation is used, arithmetic operations must be calculated twice. Therefore, number of real multiplications is $R_{M}=2 L N$ and number of real additions is $R_{A}=2(L-1) N$.

For fair comparison with DMWCST, Daubechies-4 wavelet transform will be used here. For Daubechies- $4, R_{M}=$ $8 N$, and $R_{A}=6 N$.

5.4. FFT. The FFT of $N$-points required $(N / 2) \log _{2}(N)$ complex multiplications and $N \log _{2}(N)$ complex additions [30]. Taking into consideration the fact that each complex multiplication is equal to 4 real multiplications and 2 real additions and each complex addition is equal to 2 real additions, so the computational complexity of the FFT requires $R_{M}=$ $2 N \log _{2}(N)$, and $R_{A}=3 N \log _{2}(N)$.

Table 3 shows the computational complexity for the DMWCST, oversampling DMWT, DWT, and FFT for different transform lengths $(N)$. From this table, it is clear that the DMWCST requires less computational complexity compared to oversampling DMWT and FFT, but more than DWT. However, DMWCST still compares favorably DWT because a single-level decomposition in the multiwavelet domain is equivalent to two levels in the scalar wavelet decompositions. 
TABLE 3: Comparison of computational complexity for the DMWCST, oversampling DMWT, DWT, and FFT transforms.

\begin{tabular}{lcccccccc}
\hline \multirow{2}{*}{$N$} & \multicolumn{2}{c}{ DMWCST } & \multicolumn{2}{c}{ Oversampling DMWT } & \multicolumn{2}{c}{ DWT } & \multicolumn{2}{c}{ FFT } \\
& $R_{M}$ & $R_{A}$ & $R_{M}$ & $R_{A}$ & $R_{M}$ & $R_{A}$ & 320 \\
\hline 32 & 272 & 304 & 544 & 608 & 256 & 192 & 480 \\
64 & 544 & 608 & 1088 & 1216 & 512 & 384 & 768 \\
128 & 1088 & 1216 & 2176 & 2432 & 1024 & 768 & 1792 & 2688 \\
256 & 2176 & 2432 & 4352 & 4864 & 2048 & 1536 & 4096 \\
512 & 4352 & 4864 & 8704 & 9728 & 4096 & 3072 & 9144 \\
1024 & 8704 & 9728 & 17408 & 19456 & 8192 & 6144 & 20480 \\
\hline
\end{tabular}

Thus, to get the same signal quality, the levels of computation in DMWCST are less than DWT.

\section{Conclusions}

An OFDM system based on DMWCST was proposed and compared with OFDM based on DWT and traditional OFDM based on FFT through the use of QPSK mapping technique. The performance of the systems was tested and compared in flat fading and frequency-selective fading channels. Simulation results indicated that the proposed system has very good BER performance compared to that of DWTOFDM and FFT-OFDM. Moreover, in the proposed system, the need for $\mathrm{CP}$ is dispensed with because of the excellent orthogonality that is offered by DMWCST, which subsequently reduces the system complexity, increases the transmission rate, and increases spectral efficiency. In addition, the proposed system has better BER performance, increases the bandwidth efficiency because it maintains the same data rate of the input symbols, and reduces the computational complexity compared with the oversampling DMWT-OFDM system [18]. Finally, the proposed system can be utilized as a substitute to traditional OFDM. OFDM based on DMWCST has higher bandwidth efficiency than OFDM based on FFT because of the good orthogonality of DMWCST. ISI and ICI are reduced. Thus, the use of CP in the proposed system is unnecessary.

\section{Conflict of Interests}

The authors declare that there is no conflict of interests regarding the publication of this paper.

\section{References}

[1] S. Chen and C. Zhu, "ICI and ISI analysis and mitigation for OFDM systems with insufficient cyclic prefix in time-varying channels," IEEE Transactions on Consumer Electronics, vol. 50, no. 1, pp. 78-83, 2004.

[2] S. K. Borra and S. K. Chaparala, "Performance evaluation of OFDM system with Rayleigh, Rician and AWGN channels," International Journal of Emerging Technology and Advanced Engineering, vol. 3, pp. 678-682, 2013.

[3] Y. G. Li and G. L. Stuber, Orthogonal Frequency Division Multiplexing for Wireless Communications, Springer, 2006.

[4] J. A. C. Bingham, ADSL, VDSL, and Multicarrier Modulation, John Wiley \& Sons, 2000.
[5] M. Oltean, "Wavelet OFDM performance in flat fading channels," Scientific Bulletin of University Politehnica Timisoara, ETC Series, vol. 52, pp. 167-172, 2007.

[6] S. Sengar and P. P. Bhattacharya, "Performance improvement in OFDM system by PAPR reduction," Signal \& Image Processing, vol. 3, no. 2, pp. 157-169, 2012.

[7] S. H. Han and J. H. Lee, "An overview of peak-to-average power ratio reduction techniques for multicarrier transmission," IEEE Wireless Communications, vol. 12, no. 2, pp. 56-65, 2005.

[8] A. R. Lindsey, "Wavelet packet modulation for orthogonally multiplexed communication," IEEE Transactions on Signal Processing, vol. 45, no. 5, pp. 1336-1339, 1997.

[9] B. G. Negash and H. Nikookar, "Wavelet based OFDM for wireless channels," in Proceedings of the IEEE International Conference on Vehicular Technology Conference (VTS '01), vol. 1, pp. 688-691, IEEE, Rhodes, Greece, May 2001.

[10] H. Zhang, D. Yuan, M. Jiang, and D. Wu, "Research of DFTOFDM and DWT-OFDM on different transmission scenarios," in Proceedings of the 2nd International Conference on Information Technology and Applications (ICITA '04), pp. 3-5, January 2004.

[11] K. Abdullah and Z. M. Hussain, "Performance of fourier-based and wavelet-based OFDM for DVB-T systems," in Proceedings of the Australasian Telecommunication Networks and Applications Conference (ATNAC '07), pp. 475-479, December 2007.

[12] G. Gowri, G. U. Maheswari, E. Vishnupriya, S. Prabha, D. Meenakshi, and N. R. Raajan, "Performance analysis of DWTOFDM and FFT-OFDM systems," International Journal of Engineering and Technology, vol. 5, no. 2, pp. 1455-1461, 2013.

[13] Y. J. Harbi and A. G. Burr, Comparison of Discrete Wavelet and FFT-OFDM under Different Channel Conditions, PGNet, 2014.

[14] S. Tripathi, A. Rastogi, K. Sachdeva, M. Sharma, and P. Sharma, "PAPR reduction in OFDM system using DWT with nonlinear high power amplifier," International Journal of Innovative Technology and Exploring Engineering, vol. 2, no. 5, pp. 184-188, 2013.

[15] V. Strela, P. N. Heller, G. Strang, P. Topiwala, and C. Heil, "The application of multiwavelet filterbanks to image processing," IEEE Transactions on Image Processing, vol. 8, no. 4, pp. 548$563,1999$.

[16] D. Milovanovic, A. Marincic, G. Petrovic, and Z. Barbaric, "Comparative study of scalar and multiwavelet filters in transform-based compression of IRLS images," in Proceedings of the 4th International Conference on Telecommunications in Modern Satellite, Cable and Broadcasting Services, pp. 169-172, 1999.

[17] M. B. Martin and A. E. Bell, "New image compression techniques using multiwavelets and multiwavelet packets," IEEE 
Transactions on Image Processing, vol. 10, no. 4, pp. 500-510, 2001.

[18] A. H. Kattoush, W. A. Mahmoud, and S. Nihad, "The performance of multiwavelets based OFDM system under different channel conditions," Digital Signal Processing: A Review Journal, vol. 20, no. 2, pp. 472-482, 2010.

[19] M. A. kadhim and W. Ismail, "Implementation of WiMAX (IEEE802.16.d) OFDM baseband transceiver-based multiwavelet OFDM on a multi-core software-defined radio platform," ISRN Signal Processing, vol. 2011, Article ID 750878, 9 pages, 2011.

[20] M. A. Kadhim, S. H. Farhan, and M. I. Gamaj, "Design and improvement of HiperLAN/2 physical layer model based multiwavelet signals," Innovative Systems Design and Engineering, vol. 5, no. 6, pp. 20-27, 2014.

[21] P. B. Zadeh and C. Serdean, "Multiwavelets in the context of hierarchical stereo correspondence matching techniques," International Journal on Advances in Telecommunications, vol. 4, pp. 48-57, 2011.

[22] L. A. Abdul-Rahaim and R. S. Mohammad, "Video compression using multiwavelet critically sampling transformation," Journal of Telecommunications, vol. 20, no. 2, pp. 7-13, 2013.

[23] V. Strela and A. T. Walden, "Orthogonal and biorthogonal multiwavelets for signal denoising and image compression," in Aerospace/Defense Sensing and Controls, pp. 96-107, 1998.

[24] A. Zaier and R. Bouallègue, "Channel estimation study for block-pilot insertion in OFDM systems under slowly time varying condition," International journal of Computer Networks and Communications, vol. 3, no. 6, pp. 39-54, 2011.

[25] K. Abdullah and Z. M. Hussain, "Performance of fourier-based and wavelet-based OFDM for DVB-T systems," in Proceedings of the Australasian Telecommunication Networks and Applications Conference (ATNAC '07), pp. 475-479, Christchurch, New Zealand, December 2007.

[26] M. Lallart, K. E. Nolan, P. Sutton, and L. E. Doyle, "On-the-fly synchronization using wavelet and wavelet packet OFDM," in Proceedings of 13th European Wireless Conference, Paris, France, April 2007.

[27] W. Wu, V. V. G. Srinivasan, C. Hsu, Y. Kim, C. Lee, and T. Rappaport, Wireless Communication Project (EE381K-11) Technical Report Optimal Channel Estimation for Capacity Maximization in OFDM Systems, Department of Electrical and Computer Engineering, University of Texas, Austin, Tex, USA, 2003.

[28] S. A. Ghauri, S. Alam, M. F. Sohail, A. Ali, and F. Saleem, "Implementation of OFDM and channel estimation using LS and MMSE estimators," International Journal of Computer \& Electronics Research, vol. 2, pp. 41-46, 2013.

[29] M. B. Martin, Applications of multiwavelets to image compression [M.S. thesis in Electrical Engineering], Virginia Polytechnic Institute and State University (Virginia Tech), Blacksburg, Va, USA, 1999.

[30] H. A. Leftah and S. Boussakta, "Novel OFDM based on Ctransform for improving multipath transmission," IEEE Transactions on Signal Processing, vol. 62, no. 23, pp. 6158-6170, 2014. 


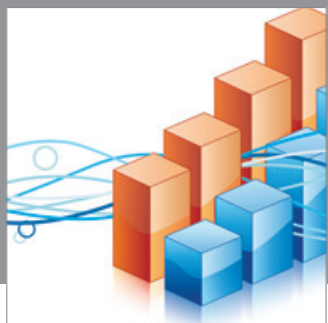

Advances in

Operations Research

mansans

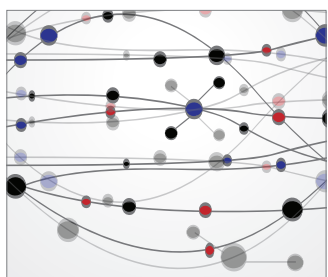

The Scientific World Journal
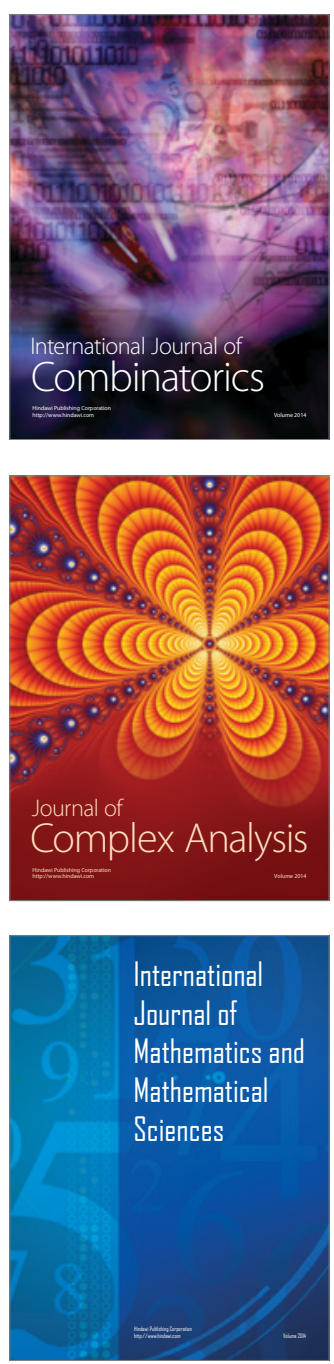
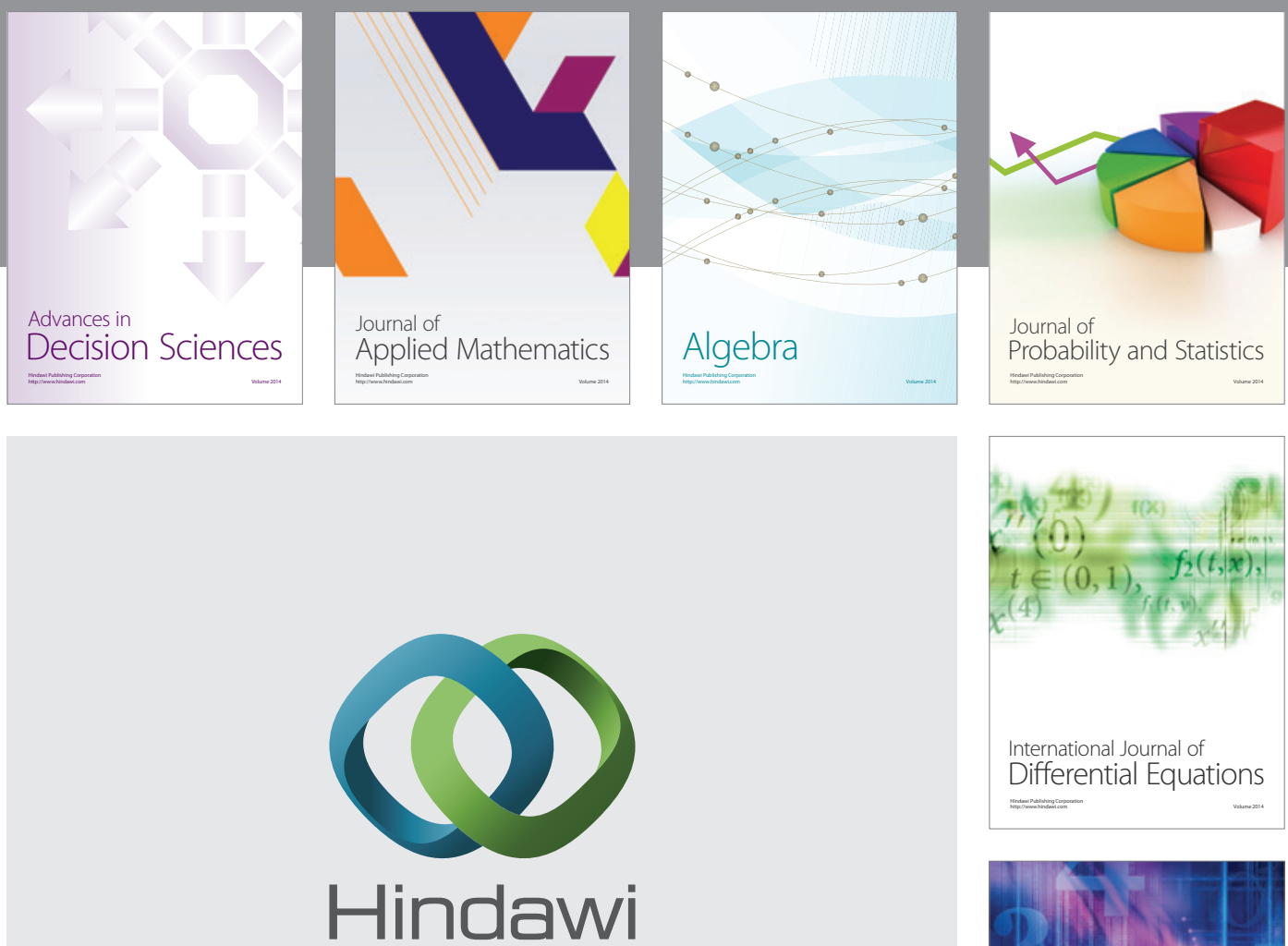

Submit your manuscripts at http://www.hindawi.com
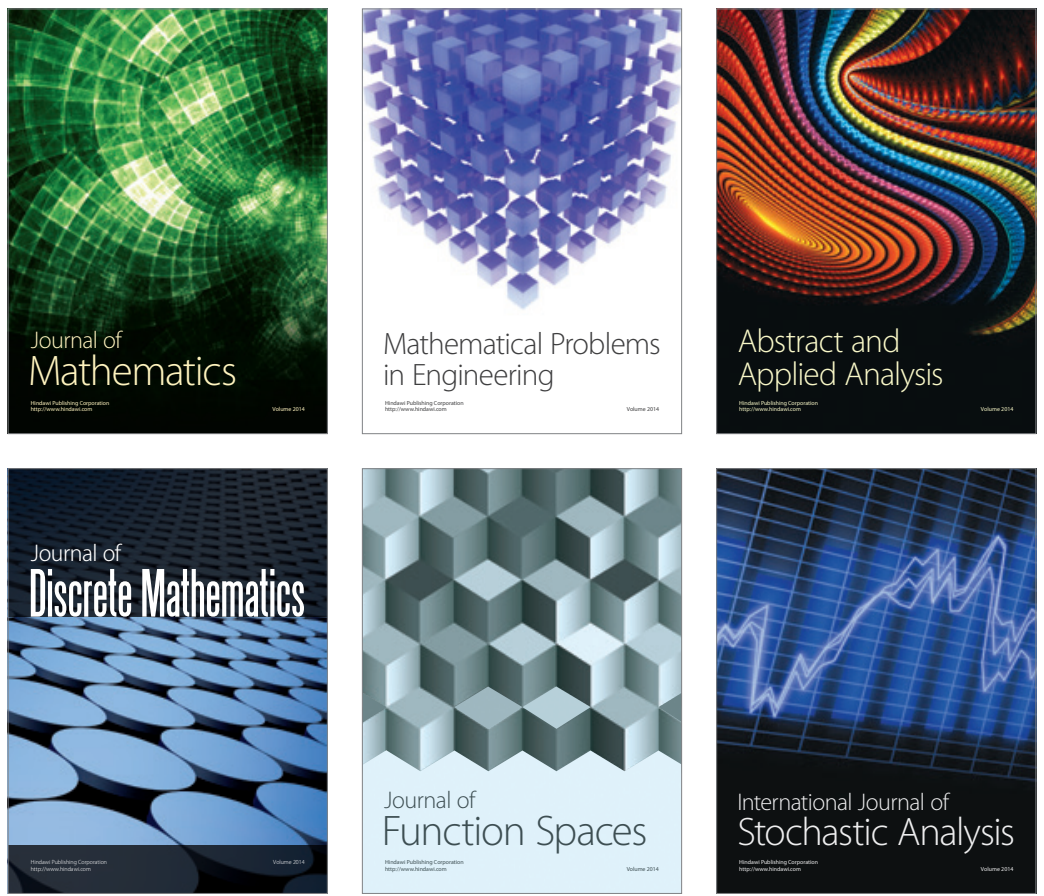

Journal of

Function Spaces

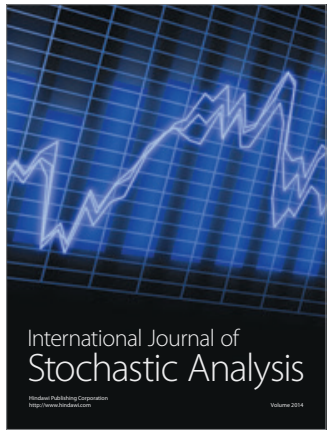

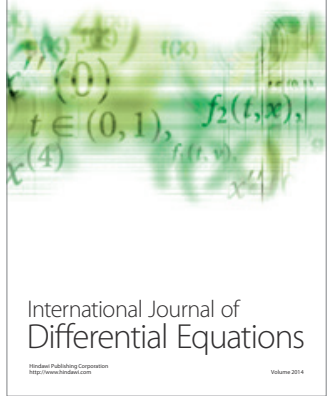
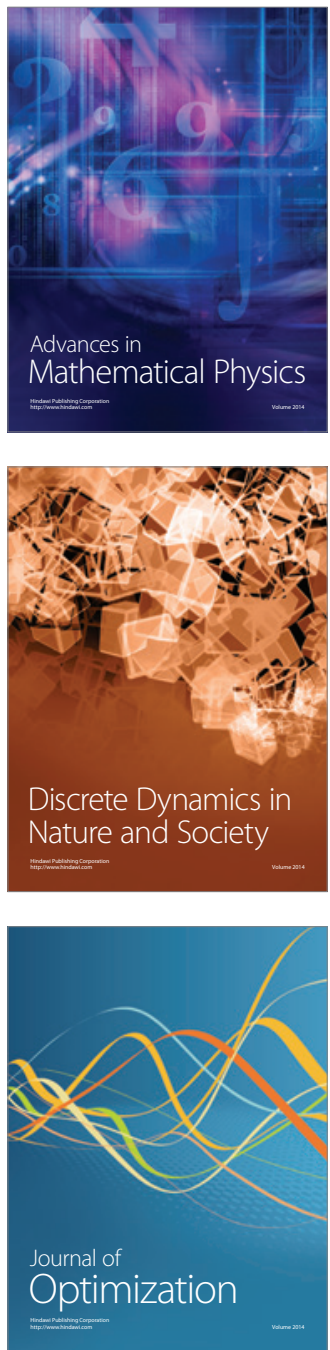